PROPOSIÇÃO DE METODOLOGIA DE TERMODEGRADAÇÃO POR MEIO DE FRAGMENTOS DE LENHOS DA Araucaria columnaris

\title{
PROPOSAL OF A THERMAL DEGRADATION METHODOLOGY WITH SCRAPS OF LOGS FROM Araucaria columnaris
}

\author{
DE LARA, Daniela ${ }^{1}$; BRESCIANI, Laís²; HILGEMANN, Maurício ${ }^{3}$; OSTERKAMP, Isa Carla ${ }^{4}$; \\ JASPER, André ${ }^{5}$ UHL, Dieter ${ }^{6}$; STÜLP, Simone ${ }^{7^{\star}}$ \\ ${ }^{1}$ Universidade Estadual do Rio Grande do Sul - Uergs, Unidade do Alto da Serra Botucaraí Soledade, Rua \\ Travessa Ticiano Felippi, 100 - Bairro Botucaraí, cep 99300-000, Soledade - RS, Brasil \\ (fone: +55 54 3381-1483) \\ ${ }^{1,2,3,7}$ Centro Universitário UNIVATES, Centro de Ciências Exatas e Tecnológicas, Rua Avelino Talini, 171 - \\ Bairro Universitário, cep 95914-014, Lajeado - RS, Brasil \\ (fone: +55 $513714-7000$ ) \\ ${ }^{4,5}$ Centro Universitário UNIVATES, Centro de Ciências Biológicas e da Saúde, Rua Avelino Talini, 171 - Bairro \\ Universitário, cep 95914-014, Lajeado - RS, Brasil \\ (fone: +55 $513714-7000$ ) \\ ${ }^{5,6}$ Senckenberg Forschungsinstitut und Naturmuseum, Senckenberganlage 25, cep 60325, Frankfurt am Main - \\ Germany. Senckenberg Center for Human Evolution and Palaeoenvironment, Institut für Geowissenschaften, \\ Universität Tübingen, cep 72076, Tübingen - Germany \\ (fone: $+55+49(0) 69 / 7542-0)$ \\ *Autor correspondente
e-mail: stulp@univates.br
}

Received 16 June 2017; received in revised form 23 August 2017; accepted 23 August 2017

\section{RESUMO}

A reconstrução de ambientes pretéritos têm sido estudo em diversas áreas da ciência objetivando auxiliar no entendimento dos diversos eventos ocorridos no planeta. As plantas são consideradas marcadores ambientais e, normalmente, mostram adaptações estruturais que são características de seu habitat. Sendo assim, após passarem pelo processo de queima, podem não ser completamente consumidas pelo fogo, sendo possível encontrar resquícios de carvão vegetal e outros materiais remanescentes. Baseado nisto, a presença de carvão vegetal se constitui em indicativo direto da ocorrência de incêndios atuais ou pretéritos, tendo contribuído com uma valiosa fonte de dados proxy (paleo)ambientais. Neste sentido, o trabalho objetiva avaliar as alterações físicas e químicas que ocorrem durante o processo de carbonização em mufla de fragmentos de lenhos da espécie Araucaria columnaris em diferentes temperaturas, permitindo avaliar influências importantes em relação ao seu processo de formação. As temperaturas da queima dos lenhos in natura foram num intervalo de 50 a $1000^{\circ} \mathrm{C}$, variando-se a temperatura de $50 \mathrm{em} 50 \stackrel{\circ}{\circ}$, com razão de aquecimento avaliada em $25{ }^{\circ} \mathrm{C} \mathrm{min}^{-1}$. A análise deste resultado busca permitir a proposição de metodologia que auxilie na caracterização dos processos naturais envolvidos na formação desse tipo de material, servindo de subsídio para inferências (paleo)ambientais.

Palavras-chave: Carbonização, Carvão Vegetal, Paleo (ambientes). 


\section{ABSTRACT}

The reconstruction of past environments has been studied in several areas of science, aiming to help in understanding the several events that occurred on the planet. Plants are considered environmental markers and usually show structural adaptations that are characteristic of their habitat. Therefore, after passing through the charring process, they may not be completely consumed by the fire, being possible to find remnants of charcoal and other remaining materials. Based on this, the presence of charcoal is an evidence of the occurrence of current or past fires, contributing with a valuable source of (paleo) environmental proxy data. In this sense, the objective of this work is to assess the physical and chemical changes that occur on scraps of logs from Araucaria columnaris. It will be done during the charring process in muffle, at different temperatures, allowing us to assess important influences in relation to its forming process. The charring temperatures of the logs in natura were in a range of 50 to $1000^{\circ} \mathrm{C}$, with ranging temperatures from 50 to $50^{\circ} \mathrm{C}$, and heating ramp of $25^{\circ} \mathrm{C} \mathrm{min}$ 1 . The analysis of this result seeks to allow the proposal of a methodology that assists in the characterization of the natural processes involved in the forming of this type of material, serving as a contribution to (paleo) environmental inferences.

Keywords: Carbonization, Charcoal, Paleo (environments).

\section{INTRODUÇÃO}

Um dos maiores desafios da humanidade constituindo-se em pontos de discussão em diferentes áreas da ciência é a modificação ocorrida nos ambientes naturais (Chamley, 2003).

Explicar essas modificações pelas quais o planeta já passou é uma das formas de se estabelecer cenários ambientais futuros, efetuando estudos de eventos pretéritos, focando em suas causas e consequências para tentar compreender o ambiente contemporâneo (Jasper e Ulh, 2011).

Sorokhtin et al. (2007) salientam que no estabelecimento de padrões para cenários futuros faz-se necessário observar como o meio ambiente evolui durante o tempo. Baseado nessas afirmações, as avaliações de fenômenos ambientais passados podem ser aplicadas na atualidade, e com isso, tornarem-se importantes ferramentas de reconstruções (paleo)ambientais.

As plantas normalmente mostram adaptações estruturais que são características de seu habitat e constituem indicadores (paleo)ambientais especialmente sensíveis (Chaloner e Mcelwain, 1997). Pires et al. (2011) salientam que as plantas são consideradas como testemunho importante acerca de eventos ambientais que afetaram e continuam afetando o planeta.

Gastaldo et al. (1996) citam que as plantas podem ser nomeadas como marcadores ambientais, e consequentemente, possibilitam a construção de um instrumento para a compreensão das variações climáticas ao longo do tempo. De acordo com o exposto, as coníferas, especificamente as espécies da família Araucariaceae, são classificadas como "fóssil vivo", uma vez que estão presentes na terra há aproximadamente 300 milhões de anos. Sendo assim, compartilham de atributos de adaptação às condições ambientais que as distinguem das plantas com flores de outros grupos de gimnospermas (Kershaw e Mcglone, 1995). Tal fato as torna especialmente valiosas para geólogos e paleobotânicos (Dutra e Stranz, 2003).

Scott e Stea (2002) consideram que o fogo, por ser um agente importante na dinâmica de um grande número de ecossistemas, deve ter atuado de forma semelhante ao longo da história da Terra. Bowman et al. (2009) e Flannigan et al. (2009) citam que as análises dos incêndios vegetacionais são importantes para avaliar as relações entre o clima, o tempo, os combustíveis e as pessoas e, assim, desempenha uma das principais fontes de interação com os ecossistemas modernos.

Estudos sobre a ocorrência dos paleoincêndios, evidenciados através de análise em carvão vegetal macroscópico, são apresentadas através de diversas pesquisas, tais como: Scott e Jones (1994); Falcon-Lang (2000); Uhl et al. (2004); Uhl et al. (2008); Uhl et al. (2010); Gutsell e Jonhson (2007); Scott (2000); Scott (2010); Jasper et al. (2008a,b); Manfroi et al. (2015); Kauffmann (2008); Kauffmann (2016). Estes estudos evidenciam a anatomia do carvão vegetal e inferem sobre as possíveis reconstruções (paleo)ambientais, podendo definir 
e orientar ações de preservação e recuperação dos ambientes atuais, contribuindo como ferramenta para compreender as questões ambientais da contemporaneidade (Scott, 2000; Uhl e Kerp, 2003; Uhl et al. 2004; Jasper et al. 2007).

Neste sentido, o objetivo do presente trabalho é avaliar as alterações físicas e químicas que ocorrem durante o processo de carbonização em mufla de fragmentos de lenhos da Araucaria columnaris em diferentes temperaturas. Além disso, considerando que as condições de carbonização serão controladas em laboratório, pretende-se utilizar as variáveis aplicadas como base para possíveis inferências (paleo)ambientais envolvidas no processo de formação dos carvões vegetais.

\section{MATERIAL E MÉTODOS}

\subsection{Identificação das amostras}

Fragmentos de lenhos da espécie Araucaria columnaris foram coletados no município de Colinas (29 $32^{\prime} 28,84^{\prime \prime} \mathrm{S}$ e $51^{\circ}$ 50' $28,35 "$ L) no Rio Grande do Sul, Brasil. Foram extraídas de espécimes abatidas por motivos diversos e as amostras foram obtidas em forma de discos a 1,50 m de altura, medindo $8 \mathrm{~cm}$ de espessura e 18 a $35 \mathrm{~cm}$ de diâmetro conforme apresentado na Figura 1.

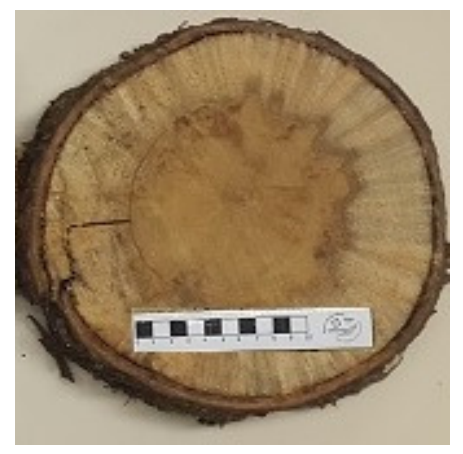

Figura 1. Amostra do fragmento de lenho do espécime Araucaria columnaris

Os lenhos secos foram identificados de duas formas diferentes, antes e depois do processo de carbonização. As amostras que antecedem a queima foram identificadas como lenhos in natura e, após o processo de queima, as amostras foram identificadas como lenhos carbonizados artificialmente.

\subsection{Processo de carbonização em mufla}

Os lenhos in natura foram carbonizados em mufla SP Labor (modelo SP-1200). As amostras foram cortadas com dimensões de 1,0 $\mathrm{cm} \times 1,0 \mathrm{~cm} \times 1,0 \mathrm{~cm}\left(1,0 \mathrm{~cm}^{3}\right)$, com variância de $0,02 \mathrm{~cm}$. Posteriormente, no cadinho de porcelana (Chiarotti A-37) foi depositada uma quantidade de areia suficiente para preencher 0 fundo do mesmo. Após essa etapa, a amostra previamente pesada foi colocada dentro do cadinho de porcelana e, objetivando restringir a quantidade de oxigênio no processo de carbonização, o recipiente foi preenchido até sua borda com areia do mar purificada (Merck), cujo tamanho de partículas variam de 0,1 a 0,3 mm.

As temperaturas da queima dos lenhos in natura foram feitas num intervalo de $50 \stackrel{\circ}{\circ}$ a $1000{ }^{\circ} \mathrm{C}$, variando-se a temperatura de 50 em 50 -C e com razão de aquecimento avaliada em 25 ${ }^{\circ} \mathrm{C} \mathrm{min}^{-1}$. A fim de se reduzir o erro experimental, todas as análises foram realizadas em triplicada determinando-se a média e o desvio padrão.

Para o processo de carbonização a temperatura de aquecimento do forno mufla foi regulada a $50 \quad{ }^{\circ} \mathrm{C}$ por 5 minutos para estabilização. Após esse tempo, as amostras foram colocadas no interior da mufla, ajustandose a temperatura desejada. Quando o equipamento atingia a temperatura estipulada, a amostra permanecia no forno por 60 minutos.

Posterior a esse período, as amostras eram retiradas da mufla e acondicionadas em dessecador para que atingissem a temperatura ambiente.

As perdas de massas durante o processo de carbonização foram calculadas a partir da massa inicial de cada amostra subtraindo-se o valor da massa final obtida e expressas em porcentagem.

\section{RESULTADOS E DISCUSSÃO:}

A Figura 3 apresenta os resultados em termos de perda de massa (\%) das amostras avaliadas em mufla para a faixa de temperatura de $50^{\circ} \mathrm{C}$ a $1000^{\circ} \mathrm{C}$ para a espécie avaliada. 


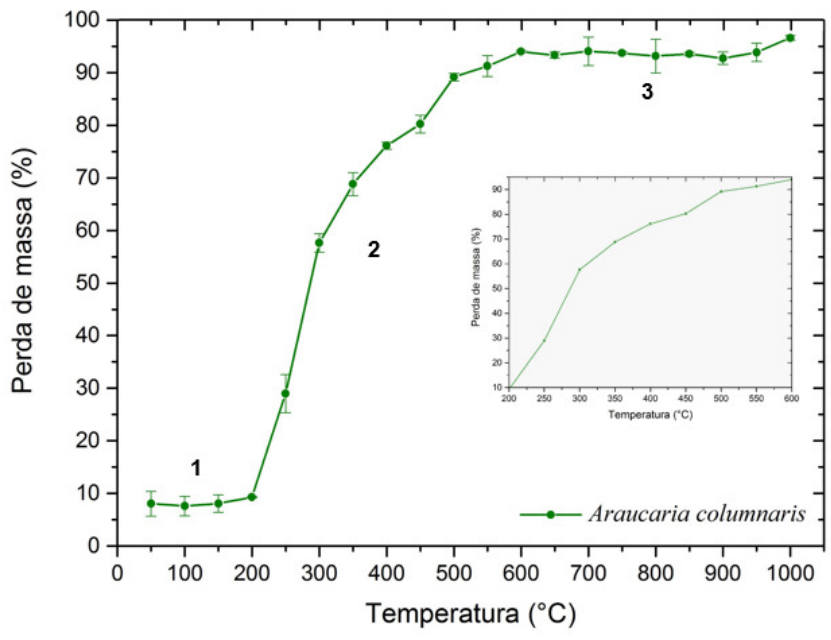

Figura 2. Perda de massa das amostras submetidas ao processo de queima em mufla para a espécie Araucaria columnaris com razão

de aquecimento de $25^{\circ} \mathrm{C} \mathrm{min}-1$ e na faixa de temperatura de $50^{\circ} \mathrm{C}$ a $1000^{\circ} \mathrm{C}$ com regiões 1,2 (detalhe em zoom) e 3 destacadas

Observa-se que a termodegradação dos lenhos ocorre em eventos térmicos definidos e padrões de queima semelhantes para as amostras avaliadas (regiões 1, 2 e 3 da Figura 2). Ainda, em destaque na mesma Figura, evidenciam-se o intervalo que houve o maior percentual de perda de massa.

Verifica-se que o intervalo de temperatura de $25{ }^{\circ} \mathrm{C}$, temperatura ambiente, até $200{ }^{\circ} \mathrm{C}$, apresenta uma região de estabilidade térmica (Fig. 2, região 1). A média da perda de massa nesta faixa de temperatura foi de 1,24\%.

A maior perda de massa se deu no intervalo de $200{ }^{\circ} \mathrm{C}$ até $600{ }^{\circ} \mathrm{C}$ (região $2 \mathrm{e} \mathrm{em}$ destaque na Figura 2). A média da perda de massa foi de $84,74 \%$ neste intervalo.

No intervalo de $600{ }^{\circ} \mathrm{C}$ até $1000 \stackrel{\circ}{\circ}$ (Fig. 2 , região 3) a perda de massa média foi de $2,61 \%$. Após a obtenção da temperatura máxima de $1000^{\circ} \mathrm{C}$, a massa residual, em termos percentuais, foi de $3,27 \%$.

As mudanças na coloração dos lenhos podem ser avaliadas na Figura 3.
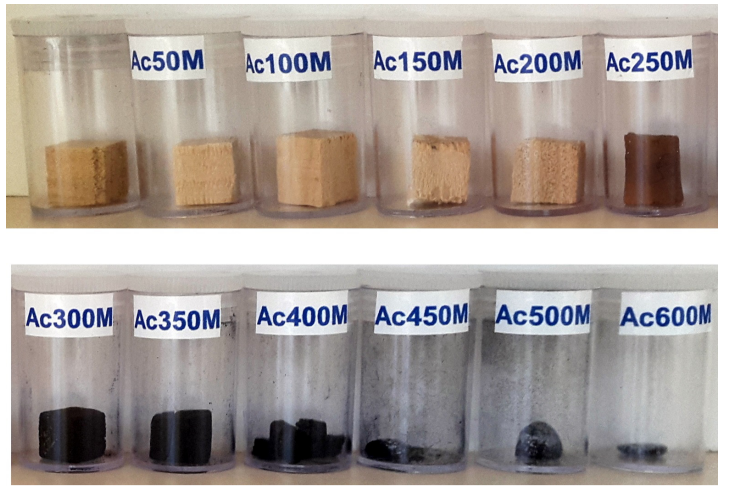

Figura 3. Mudança de coloração da amostra in natura e das amostras submetidas ao processo de queima em mufla para a espécie Araucaria columnaris na faixa de temperatura de $200^{\circ} \mathrm{C} \mathrm{a}$ $600^{\circ} \mathrm{C}$

Visualmente não se observou modificação em relação tamanho, forma e cor nas amostras carbonizadas nas temperaturas de $50{ }^{\circ} \mathrm{C}$ a 150 C. Concomitante com 0 incremento de temperatura houve diminuição do formato das amostras e as mesmas tornaram mais frágeis e consequentemente, mais quebradiças.

Rendeiro et al. (2008) citam que ao reduzir o teor de umidade das amostras, inicia-se - processo de decomposição da estrutura molecular dos lenhos. A umidade da biomassa provém da água, que faz parte da composição da estrutura macro do lenho e está ligada às paredes de celulose. A perda dessa água causa tensão na estrutura molecular e se manifesta macroscopicamente pela mudança da sua coloração para preto conforme observado na Figura 2.

A perda de massa observada na Figura 2 pode estar associada à desagregação dos principais componentes estruturais dos lenhos. Oliveira et al. (1982) citam que os lenhos quando submetidos à ação do calor, em temperaturas elevadas sofrem um processo de transformação, no qual, todos os seus componentes são exaustivamente modificados. Ainda Figueroa e Moraes (2009) citam que o lenho, por ser um polímero natural, quando submetido ao fogo, sofre despolimerização e carbonização, alterando suas propriedades mecânicas conforme o nível de temperatura e tempo de exposição ao calor ao qual foi submetido.

Beaumont (1985) cita quais as principais modificações químicas ocorridas no lenho por faixa de temperatura. $\mathrm{Na}$ fase inicial de aquecimento, entre $20{ }^{\circ} \mathrm{C}$ e $110{ }^{\circ} \mathrm{C}$, o lenho 
absorve calor e libera vapor d'agua (Fig. 2, região 1). Com o aumento da temperatura até $270^{\circ} \mathrm{C}$, o lenho começa a degradar e, ainda existem resquícios remanescentes de água que continuam sendo liberados juntamente com compostos, tais como: monóxido de carbono, dióxido de carbono, ácido acético e metanol.

A maior perda de massa durante o processo de termodegradação dos lenhos acontece na região 2 da Figura 2. Beaumont (1985) salienta que na faixa de $270 \stackrel{\circ}{\circ}$ a $290 \stackrel{\circ}{\circ}$, inicia-se o processo exotérmico, e de $290^{\circ} \mathrm{C}$ a $400{ }^{\circ} \mathrm{C}$, o lenho continua degradando-se e liberando gases combustíveis e vapores condensáveis.

Observa-se que após a temperatura atingir $600{ }^{\circ} \mathrm{C}$ (Fig. 2, região 3), acontece uma gradual diminuição na perda de massa.

Para o entendimento das alterações físico-químicas ocorridas nos lenhos após o processo de carbonização, Treusch et al. (2004) citam que deve ser observado os três principais constituintes dos lenhos: celulose, hemicelulose e lignina. A combinação de celulose (40-45\%) e a hemicelulose (15-25\%), conhecida como holocelulose, geralmente representa $65-70 \%$ do peso seco dos lenhos (Rowell et al. 2005).

Werner et al. (2014) citam que a decomposição térmica da hemicelulose variou para taxas máximas de perda de massa entre $243{ }^{\circ} \mathrm{C}$ e $332 \circ \mathrm{C}$. Já Yang et al. (2007) citam que sua faixa de decomposição está entre $220{ }^{\circ} \mathrm{C}$ e $315^{\circ} \mathrm{C}$, com perda de massa próxima a $268^{\circ} \mathrm{C}$. Já a decomposição térmica da celulose apresentou para Yang et al. (2007) e Shen et al. (2010) uma estabilidade térmica até $300^{\circ} \mathrm{C}$.

Conesa et al. (1995) citam que cada fração dos componentes dos lenhos tem uma cinética de decomposição térmica bem diferenciada e, a partir do fornecimento de altas temperaturas promove a decomposição térmica dos seus componentes químicos.

Yang et al. (2007) ressaltam que o processo de decomposição da lignina acontece lentamente desde o início da carbonização dos demais constituintes até $900{ }^{\circ} \mathrm{C}$, com baixa taxa de perda de massa. Sua degradação acontece numa faixa ampla de temperatura e apresenta maior estabilidade térmica devido a sua aromaticidade, ao tamanho e arranjo na estrutura molecular (Haykiri-Acma et al. 2010).

Característica esta oposta da celulose e da hemicelulose que possuem um arranjo estrutural mais simples e temperatura de degradação abaixo de $350{ }^{\circ} \mathrm{C}$, conforme já exposto anteriormente.

\section{CONCLUSÕES:}

Os resultados obtidos nesse estudo evidenciaram que é possível avaliar as alterações físicas e químicas que ocorrem durante o processo de carbonização em mufla de fragmentos de lenhos da Araucaria columnaris em diferentes temperaturas, permitindo avaliar a sua influência sobre características químicas dos carvões vegetais. Assim, a análise destes dados busca auxiliar na proposição de uma metodologia que auxilie na caracterização dos processos naturais envolvidos na formação desse tipo de material, servindo de subsídio para inferências (paleo)ambientais.

\section{AGRADECIMENTOS:}

Os autores agradecem aos técnicos Me. M. B. Horn e Dr. V. Ilha e à CAPES pelos auxílios financeiros concedidos, projetos CAPES (A072/2013), CNPq (301585/2012-1, 400972/2013-1, 444330/2014-3, 310421/2013-6).

A. Jasper agradece à FAPERGS, ao CNPq (305436/2015-5), à CAPES (Brasil - 8107-14-9) $e$ à Alexander von Humboldt Foundation (Germany BRA 1137359 STPCAPES).

\section{REFERÊNCIAS:}

1. Chamley, H. Geoscience, enviromenment and man. v. 1. Amsterdam: Elsevier, 2003.

2. Jasper, A.; Uhl, D. In: Goal Meeting. Freiberg: University Freiberg, v C538. p. 95-99. 2011.

3. Sorokhtin, O. G.; Chilingar, G. V.; Khilyur, L. F. In: Developments in Earth \& Environmental Sciences 5. Elsevier, 2007. 313 p. (http://lynuhec.ru/zapux.pdf).

4. Chaloner, W. G.; Mcelwain, J. Review of Palaeobotany and Palynology, 1997, 95.

5. Pires, E. F.; Guerra-Sommer, M.; Scherer, C. M. S.; Dos Santos, A. R.; Cardoso, E. Journal of South American Earth Sciences, 2011, 32, 1.

6. Gastaldo, R. A.; Dimichele, W. A.; Pfefferkorn, H. W. GSA, 1996, 6.

7. Kershaw, P.; Mcglone, M.S. The 
Quaternary history of the southern conifers. Palo Alto, California: See Enright \& Hill, 1995.

(https://www.amazon.ca/Ecology-

Southern-Conifers-Neal-

Enright/dp/toc/1560986174).

8. Dutra, T.; Stranz, A. In: Ronchi, L.H.; Coelho, O.G.W. (org.) Tecnologia diagnóstico e planejamento ambiental. São Leopoldo: Unisinos, 2003. p. 293351.

9. Scott, A.C., Stea, R. Geoscientist, 2002, 12.

10. Bowman, D.M.J.S.; Balch, J.K.; Artaxo, P.; Bond, W.J.; Carlson, J.M.; Cochrane, M.A.; D'antonio, Cm.M.; Defries, R.S.; Doyle, J.C.; Harrison, S.P.; Johnston, F.H.; Keeley, J.E.; Krawchuk, M.A.; Kull, C.A.; Marston, J.B.; Moritz, M.A.; Prentice, I.C.; Roos, C.I.; Scott, A.C.; Swetnam, T.W.; Van Der Werf, G.R.; Pyne, S.J. Science, 2009, 324.

11. Flannigan, M. D.; Krawchuk, M. A.; De Groot, W. J.; Wotton, B. M.; Gowman, L. M. Int J Wildl Fire, 2009, 18.

12. Scott, A. C.; Jones, T. P. Palaeogeography, Palaeoclimatology, Palaeoecology, 1994, 106.

13. Falcon-Lang, H. J. Palaeogeography, Palaeoclimatology, Palaeoecology, 2000, 164.

14. Uhl, D., Lausberg, S., Noll, R., Stapf, K.R.G. Palaeo, 2004, 207.

15. Uhl, D.; Jasper, A.; Hamad, A. M. B. A.; Montenari, M. 1 ${ }^{\text {a }}$ WSEAS International Conference on Environmental and Geological Science and Engineering (EG'08) Malta, Sep. 2008. p. 11-13.

16. Uhl, D., Jasper, A., Schindler, T., Wuttke, M. Palaios, 2010, 25.

17. Gutsell, S.L., Jonhson, E.A. Wildfire and Tree Population Processes. In: Jonhson, E.A., Mianishi, K. Plant Disturbance Ecology. Amsterdam: Elsevier, 2007. (https://www.elsevier.com/books/plantdisturbance-ecology/johnson/978-0-12088778-1).

18. Scott, A.C. Palaeo, 2000, 164.

19. Scott, A.C. Palaeogeography, Palaeoclimatology, Palaeoecology, 2010, 291.

20. Jasper, A., Guerra-Sommer, M., Menegat, R., Cazzullo-Klepzig, M., Branco, F.S.R.T., Salvi, J. In: Winge, M., Schobbenhaus, C., Souza, C.R.G., Fernandes, A.C.S., Berbert, B., Queiroz,
E.T.

(edit.)

2008a.

(http://www.unb.br/ig/sigep/sitio008/sitio00 8/pdf).

21. Jasper, A., Ulh, D., Guerra-Sommer, M., Mosbrugger, V. Journal of South Américan Earth Sciences, 2008b, 26.

22. Manfroi, J., Uhl, D., Guerra-Sommer, M., Francischin, H., Martinelli, A.G., Soares, M.B. \& Jasper, A. Palaeogeography, Palaeoclimatology, Palaeoecology, 2015, 436.

23. Kauffmann, M. Fragmentos de charcoal (carvão vegetal fóssil) como indicativo da ocorrência de incêndios vegetacionais durante o Quaternário do Planalto das Araucárias, Rio Grande do Sul, Brasil. 2008. Dissertação (Mestrado).

24. Kauffmann, M., Jasper, A., Uhl, D., Meneghini, J. Elsevier Science, 2016, 450.

25. Scott, A. C. Palaeogeography, Palaeoclimatology, Palaeoecololgy, 2000, 164.

26. Uhl, D.; Kerp, H. Palaeo, 2003, 199.

27. Uhl, D.; Lausberg, S.; Noll, R.; Stapf, K.R.G.

Palaeogeography, Palaeoclimatology, Palaeoecology, 2004, 207.

28. Jasper, A.; Guerra-Sommer, M.; Uhl, D.; Salvi, J.; Kauffmann, M.; Osterkamp, I. C.; Gonçalves, C. V. In: Carvalho, I. S.; Cassab, R. C. T.; Schwanke, C.; Carvalho, M. A.; Fernandes, A. C. S.; Rodrigues, M. A. C.; Carvalho, M. S. S.; Araí, M.; Oliveira, A. E. Q. O. (eds.). Paleontologia: Cenários de Vida. Rio de Janeiro: Interciência, 2007. p. 14-25.

29. Rendeiro, G., Nogueira, M., Brasil, A.C.M., Cruz, D.O.A., Guerra, D.R.S., Macêdo, E.N., Ichihara, J.A. Combustão e gasificação de biomassa sólida. Brasília: Ministério de Minas e Energia,2008.

30. Oliveira, J.B., Gomes, P.A., Almeida, M.R. Carvão vegetal: destilação, carvoejamento, propriedades, controle de qualidade. Belo Horizonte: FCTMG, 1982.

31. Figueroa, M.J.M., Moraes, P.D. Ambiente Construído, 2009, 9, 4.

32. Beaumont, E. (Ed.) FAO Forestry Paper, 1985 , 63. (http://www.fao.org/ docrep/X5555E/x555 5e00.htm\#Contents).

33. Treusch, O., Hofenauer, A., Troger, E.F., Fromm, J., Wegener, G. Wood Sci Technol, 2004, 38.

34. Rowell, R.M., Petterson, R., Han, J.S., 
Rowell, J.S., Tschabalala, M.A. Handbook of Wood Chemistry and Wood Composites, 2005.

35. Werner, K., Pommer, L., Brostrom, M. Journal of Analytical and Applied Pyrolysis, 2014, 110.

36. Yang, H., Yan, R., Chen, H., Lee, D.O., Zheng, C. Fuel, 2007, 86.

37. Shen, D.K., Gu, S., Bridgwater, A.V. Carbohydrate Polymers, 2010, 82.

38. Conesa, J.A., Caballero, J.A., Marcilla, A., Font, R. Thermochimica Acta, 1995, 254.

39. Yang, H., Yan, R., Chen, H., Li, D.H., Zheng, C. ScienceDirect, 2007, 86.

40. Haykiri-Acma, H., Yaman, S., Kucukbayrak, S. Fuel Processing Technology, 2010, 91. 